Karel Kolman (Praha)

\title{
SUPERCONVERGENCE BY STEKLOV AVERAGING IN THE FINITE ELEMENT METHOD
}

Abstract. The Steklov postprocessing operator for the linear finite element method is studied. Superconvergence of order $\mathcal{O}\left(h^{2}\right)$ is proved for a class of second order differential equations with zero Dirichlet boundary conditions for arbitrary space dimensions. Relations to other postprocessing and averaging schemes are discussed.

1. Introduction. Most finite element postprocessing schemes for second order problems discretized on simplicial partitions of domains appear in the form of gradient postprocessing, i.e., an improved approximation of the gradient of the true solution is obtained, but the resulting vector function is not a potential field.

Steklov averaging based postprocessing proposed by Oganesyan and Rukhovets in [6] does not suffer from this disadvantage.

The monograph [6] (pp. 94-101, 189) presents the Steklov operator $S_{h}$ (which will be defined in Section 2) as a tool for postprocessing of finite element solutions of second order elliptic boundary value problems. Fixing a subdomain $\Omega_{0}$ with $\bar{\Omega}_{0} \subset \Omega$, and taking the finite element approximate solution $u_{h}$ of the Poisson problem $-\Delta u=f$ with zero Dirichlet boundary condition, Oganesyan and Rukhovets proved the error bound

$$
\left\|u-S_{h} u_{h}\right\|_{1, \Omega_{0}}=\mathcal{O}\left(h^{3 / 2}\right)
$$

for 2-dimensional uniform meshes consisting of right isosceles triangles, whereas the piecewise linear finite element solution $u_{h}$ approximates the exact solution $u$ with order

$$
\left\|u-u_{h}\right\|_{1, \Omega}=\mathcal{O}(h) .
$$

2000 Mathematics Subject Classification: Primary 65N30.

Key words and phrases: superconvergence, finite elements, postprocessing, averaging. 
Numerical experiments, however, suggest that bound (1) is not optimal, i.e., it may be improved.

In this paper we show that, in fact, for $u \in H^{3}(\Omega)$ and uniform meshes we have

$$
\left\|u-S_{h} u_{h}\right\|_{1, \Omega_{0}} \leq C h^{2}\|u\|_{3, \Omega}
$$

for some $C>0$.

2. Steklov averaging operator. Let $\Omega_{0}$ with $\bar{\Omega}_{0} \subset \Omega \subset \mathbb{R}^{d}, d \geq 1$, be a subdomain of a bounded domain $\Omega$. The $d$-dimensional Steklov averaging operator acting on a function $v \in L^{1}(\Omega)$ is defined by

$$
\left(S_{h} v\right)(x)=\frac{1}{(2 h)^{d}} \int_{\Pi_{h}} v(x+y) d y
$$

where $\Pi_{h}=\left\{y=\left(y_{1}, \ldots, y_{d}\right) \in \mathbb{R}^{d}|| y_{i} \mid \leq h, i=1, \ldots, d\right\}, x \in \Omega_{0}$, and $h$ is small enough so that $x+\Pi_{h} \subset \Omega$.

Lemma 2.1. For each integer $l \geq 0$ and a fixed subdomain $\Omega_{0}$ with $\bar{\Omega}_{0} \subset \Omega$ there exist constants $C>0$ and $h_{0}>0$ such that for all $h \in\left(0, h_{0}\right)$,

$$
\left\|S_{h} v\right\|_{l, \Omega_{0}} \leq C\|v\|_{l, \Omega} \quad \forall v \in H^{l}(\Omega)
$$

and

$$
\left\|v-S_{h} v\right\|_{1, \Omega_{0}} \leq C h^{2}\|v\|_{3, \Omega} \quad \forall v \in H^{3}(\Omega) .
$$

Proof. Oganesyan and Rukhovets' monograph [6] contains an elementary proof of (3) and the bound

$$
\left\|v-S_{h} v\right\|_{0, \Omega_{0}} \leq C h^{2}\|v\|_{2, \Omega}
$$

for $d=2$ (pp. 94-96). Bramble and Schatz's paper [1] includes the bounds (3) and (5) in Lemmas 5.2 and 5.3 for arbitrary space dimensions (take $S_{h} v=K_{h, l}^{2 t} * v$ with $t=1$ and $\left.l=1\right)$.

If we make use of the equality $S_{h} \nabla v=\nabla S_{h} v$ proved in Lemma 2.2 below, where the Steklov operator for vector functions is naturally defined by

$$
\left(S_{h} \nabla v\right)_{i}=S_{h} \frac{\partial}{\partial x_{i}} v, \quad i=1, \ldots, d,
$$

inequality (5) immediately implies (4).

Lemma 2.2. If $v \in H^{1}(\Omega)$, then

$$
S_{h} \nabla v=\nabla S_{h} v
$$

in $\Omega_{0}$ and

$$
S_{h} v \in C^{1}\left(\bar{\Omega}_{0}\right) .
$$


Proof. For a function $\varphi \in C_{0}^{\infty}\left(\Omega_{0}\right)$, by the Fubini theorem and the definition of the distributional derivative we have

$$
\begin{aligned}
\left(S_{h} \partial_{i} v, \varphi\right)_{\Omega_{0}} & =\frac{1}{(2 h)^{d}} \int_{\Omega_{0}}\left(\int_{\Pi_{h}} \partial_{i} v(x+y) d y\right) \varphi(x) d x \\
& =\frac{1}{(2 h)^{d}} \int_{\Pi_{h}}\left(\int_{\Omega_{0}} \partial_{i} v(x+y) \varphi(x) d x\right) d y \\
& =-\frac{1}{(2 h)^{d}} \int_{\Pi_{h}}\left(\int_{\Omega_{0}} v(x+y) \partial_{i} \varphi(x) d x\right) d y \\
& =-\frac{1}{(2 h)^{d}} \int_{\Omega_{0}}\left(\int_{\Pi_{h}} v(x+y) d y\right) \partial_{i} \varphi(x) d x=-\left(S_{h} v, \partial_{i} \varphi\right)_{\Omega_{0}}
\end{aligned}
$$

and the distributional derivative $\left(\partial / \partial x_{i}\right) S_{h} v$ is, therefore, a function from $L^{2}\left(\Omega_{0}\right)$ and equals $S_{h}\left(\partial / \partial x_{i}\right) v$.

For a function $v \in L^{2}(\Omega)$ we get $S_{h} v \in C\left(\bar{\Omega}_{0}\right)$, since

$$
\left(S_{h} v\right)(x)-\left(S_{h} v\right)(y)=\frac{1}{(2 h)^{d}} \int_{\Upsilon_{h}(x, y)} v(\xi) d \xi \leq \frac{1}{(2 h)^{d}}\|1\|_{L^{2}\left(\Upsilon_{h}(x, y)\right)}\|v\|_{L^{2}(\Omega)},
$$

where the set $\Upsilon_{h}(x, y)$ is defined by

$$
\Upsilon_{h}(x, y)=\left(\left(x+\Pi_{h}\right) \cup\left(y+\Pi_{h}\right)\right) \backslash\left(\left(x+\Pi_{h}\right) \cap\left(y+\Pi_{h}\right)\right)
$$

(see Figure 1) and apparently

$$
\lim _{x \rightarrow y}\|1\|_{L^{2}\left(\Upsilon_{h}(x, y)\right)}=0
$$

for a fixed $h$.

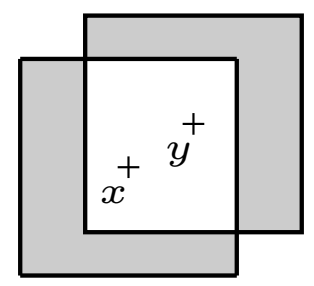

Fig. 1. $\Upsilon_{h}(x, y)$ for $d=2$ (shaded area)

Applying the above continuity property to $S_{h} \nabla u$, we get $S_{h} u \in C^{1}\left(\bar{\Omega}_{0}\right)$.

REMARK 2.3. Clearly, (2) can be rewritten as

$$
S_{h} v=\frac{1}{(2 h)^{d}} v * \chi_{h},
$$

where $\chi_{h}$ is the characteristic function of the set $\Pi_{h}$ and we extend the function $v$ in some way to $L^{1}\left(\mathbb{R}^{d}\right)$. 
EXAmPle 2.4. For $v(x)=\sin (\pi x), x \in \mathbb{R}^{1}$, we obtain

$$
\left(S_{h} v\right)(x)=\frac{1}{2 h} \int_{x-h}^{x+h} \sin \pi y d y=\frac{\sin \pi h}{\pi h} \sin \pi x
$$

and

$$
\left(S_{h} v\right)^{(n)}(x)=\left(S_{h} v^{(n)}\right)(x)=\frac{1}{2 h} \int_{x-h}^{x+h} \sin ^{(n)} \pi y d y=\frac{\sin \pi h}{\pi h} \sin ^{(n)} \pi x
$$

for the $n$th derivative.

3. Steklov averaging in FEM. We shall consider second order elliptic boundary value problems of the type

$$
\begin{aligned}
-\operatorname{div}(\mathbb{A}(x) \nabla u(x)) & =f(x) & & \text { in } \Omega, \\
u(x) & =0 & & \text { on } \partial \Omega,
\end{aligned}
$$

where the matrix $\mathbb{A}(x)$ is uniformly positive definite and has a bounded total derivative, and the right-hand side $f(x)$ and $\Omega$ are such that the conditions of the so-called supercloseness (see [2] and Section 3.1 below) of the piecewise linear finite element solution $u_{h}$ to the piecewise linear nodal Lagrange interpolant $L_{h} u$ are satisfied.

3.1. Uniform simplicial partitions. In the following we suppose that the finite element solution $u_{h}$ is computed on a $d$-simplicial partition $\mathcal{T}_{h}=\left\{\tau_{h, i}\right\}_{i}$ of $\bar{\Omega}$ (which is a bounded polytopic domain). We further assume that the partition is constructed by translation of a partition of the $d$-hypercube $K_{h}^{d}$ with edge size $h$ into $d ! d$-simplices,

$$
S_{\sigma, h}=\left\{x=\left(x_{1}, \ldots, x_{d}\right) \in \mathbb{R}^{d} \mid 0 \leq x_{\sigma(1)} \leq \cdots \leq x_{\sigma(d)} \leq h\right\},
$$

where $\sigma$ denotes a permutation of $1, \ldots, d$ and $\sigma(j)$ its $j$ th component.

The details of the construction and properties of the partition are presented in [2]. The partition constructed has internal vertices and midpoints of internal edges of the $d$-simplices as symmetry points (see Theorem 2.5 in $[2])$. We call a partition uniform if for each internal edge the union of simplices sharing the edge is a point-symmetric set with respect to the midpoint of the edge.

An important property of the Galerkin solution $u_{h}$ computed on partitions of this type is its so-called supercloseness to $L_{h} u$. It is described by the bound

$$
\left\|L_{h} u-u_{h}\right\|_{1, \Omega} \leq C h^{2}\|u\|_{3, \Omega}
$$

(see [2]). Here $L_{h} u$ is the piecewise linear Lagrange interpolation of $u$ at the vertices of the simplices of the partition. 
3.2. Superconvergence bound. The main goal of this paper is to prove the following theorem.

TheOREM 3.1. Let $\Omega_{0}$ with $\bar{\Omega}_{0} \subset \Omega \subset \mathbb{R}^{d}, d \geq 1$, be a subdomain of a bounded domain $\Omega$. Suppose that a regular family of uniform partitions is used in the discretization and that $u \in H^{3}(\Omega)$. Then there exists a constant $C>0$ such that

$$
\left\|u-S_{h} u_{h}\right\|_{1, \Omega_{0}} \leq C h^{2}\|u\|_{3, \Omega}
$$

for $h$ small enough.

In the following paragraphs we will be working with the nodal linear Lagrange interpolant $L_{h} u$ of the true solution. To guarantee that $L_{h} u$ is well defined we shall suppose that $u \in H^{s}(\Omega)$ with $s=3$ when $d \leq 5$ and $s>d / 2$ when $d \geq 6$. This assumption is not an obstacle to proving the superconvergence property for $u \in H^{3}(\Omega)$, which is obtained by a density argument.

By the triangle inequality

$$
\begin{aligned}
\left\|u-S_{h} u_{h}\right\|_{1, \Omega_{0}} \leq & \left\|u-S_{h} L_{h} u\right\|_{1, \Omega_{0}}+\left\|S_{h}\left(L_{h} u-u_{h}\right)\right\|_{1, \Omega_{0}} \\
\leq & \left\|u-S_{h} u\right\|_{1, \Omega_{0}}+\left\|S_{h}\left(u-L_{h} u\right)\right\|_{1, \Omega_{0}} \\
& +\left\|S_{h}\left(L_{h} u-u_{h}\right)\right\|_{1, \Omega_{0} .}
\end{aligned}
$$

We can use (4) to bound the first term on the right-hand side of (11),

$$
\left\|u-S_{h} u\right\|_{1, \Omega_{0}} \leq C h^{2}\|u\|_{3, \Omega} .
$$

Using the supercloseness property (9) of the finite element solution $u_{h}$ and boundedness (3) of $S_{h}$, we get

$$
\left\|S_{h}\left(L_{h} u-u_{h}\right)\right\|_{1, \Omega_{0}} \leq C h^{2}\|u\|_{3, \Omega} .
$$

The boundedness of $S_{h}$ and approximation theory (see [3]) imply the $L^{2}$ bound

$$
\left\|S_{h}\left(u-L_{h} u\right)\right\|_{0, \Omega_{0}} \leq C h^{2}\|u\|_{3, \Omega},
$$

and therefore, it remains to estimate the term

$$
\left|S_{h}\left(u-L_{h} u\right)\right|_{1, \Omega_{0}} .
$$

3.3. One-dimensional case. In the one-dimensional case, we have

$$
\left|S_{h}\left(u-L_{h} u\right)\right|_{1, \Omega_{0}}=\left\|\left(S_{h}\left(u-L_{h} u\right)\right)^{\prime}\right\|_{0, \Omega_{0}}=\left\|S_{h}\left(u^{\prime}-\left(L_{h} u\right)^{\prime}\right)\right\|_{0, \Omega_{0}} .
$$

For any node $N_{i}$ of a given uniform partition of the interval $\Omega$ we obtain

$$
\left(S_{h} u^{\prime}\right)\left(N_{i}\right)=\frac{1}{2 h} \int_{N_{i-1}}^{N_{i+1}} u^{\prime}(x) d x=\frac{u\left(N_{i+1}\right)-u\left(N_{i-1}\right)}{2 h}
$$


(the central difference derivative approximation at $N_{i}$ ) and

$$
\begin{aligned}
\left(S_{h}\left(L_{h} u\right)^{\prime}\right)\left(N_{i}\right) & =\frac{1}{2 h}\left[u\left(N_{i+1}\right)-u\left(N_{i}\right)+u\left(N_{i}\right)-u\left(N_{i-1}\right)\right] \\
& =\frac{u\left(N_{i+1}\right)-u\left(N_{i-1}\right)}{2 h} .
\end{aligned}
$$

Since $S_{h}\left(L_{h} u\right)^{\prime}$ is piecewise linear (on the same partition of $\Omega$ ), we get

$$
S_{h}\left(L_{h} u\right)^{\prime}=L_{h} S_{h} u^{\prime},
$$

and consequently,

$$
\left|S_{h}\left(u-L_{h} u\right)\right|_{1, \Omega_{0}}=\left\|\left(I-L_{h}\right) S_{h} u^{\prime}\right\|_{0, \Omega_{0}} \leq C h^{2}\left\|S_{h} u^{\prime}\right\|_{2, \Omega_{0}} \leq C h^{2}\|u\|_{3, \Omega},
$$

which is the required bound.

3.4. Properties of $S_{h} L_{h} u$ and $S_{h} \nabla L_{h} u$. The situation for $d>1$ is more complicated. We define

$$
V_{h}^{k}(\Omega)=\left\{v \in C(\bar{\Omega})|v|_{\tau_{h}} \in P^{k}\left(\tau_{h}\right) \forall \tau_{h} \in \mathcal{T}_{h}\right\},
$$

where $P^{k}\left(\tau_{h}\right)$ is the space of polynomials of degree $k$ on a $d$-simplex $\tau_{h}$.

Lemma 3.2. If $v_{h} \in V_{h}^{1}(\Omega)$, then

$$
\begin{aligned}
S_{h} \nabla v_{h} & \in\left(V_{h}^{d}\left(\Omega_{0}\right)\right)^{d}, \\
S_{h} v_{h} & \in V_{h}^{1+d}\left(\Omega_{0}\right) .
\end{aligned}
$$

Proof. The function $\partial v_{h} / \partial x_{i}$ is constant on each $\tau_{h} \in \mathcal{T}_{h}$ for $i=1, \ldots, d$. From the structure of the simplicial mesh defined in Section 3.1 (see the definition of $S_{\sigma, h}$ in Section 3.1 and the definition of $\Pi_{h}$ in Section 2), we clearly see that for a fixed simplex $\tau_{h}$, the function $f_{\tau_{h}}(x)=$ meas $\left(\left(x+\Pi_{h}\right) \cap \tau_{h}\right)$ is a piecewise polynomial function in $V_{h}^{d}\left(\Omega_{0}\right)$. The inclusion (14) is thus proved. Inclusion (15) then follows from the equality $S_{h} \nabla v_{h}=\nabla S_{h} v_{h}$

For $d=1$ the smoothed function $S_{h} L_{h} u$ is thus piecewise quadratic $\left(S_{h} \nabla L_{h} u\right.$ is piecewise linear).

For $d=2$ the smoothed function $S_{h} L_{h} u$ is piecewise cubic $\left(S_{h} \nabla L_{h} u\right.$ is piecewise quadratic).

For $d=3$ the smoothed function $S_{h} L_{h} u$ is piecewise quartic $\left(S_{h} \nabla L_{h} u\right.$ is piecewise cubic).

3.5. Quadratic polynomial gradient recovery at symmetry points. For $N \in \bar{\Omega}_{0}$ define

$$
F_{i, N}(u)=S_{h} \frac{\partial}{\partial x_{i}}\left(u-L_{h} u\right)(N)=\frac{1}{(2 h)^{d}} \int_{\Pi_{h}} \frac{\partial}{\partial x_{i}}\left(u-L_{h} u\right)(N+y) d y .
$$

Let us now take $N$ to be a local symmetry point of the partition, by which we mean that for any continuous function $v_{\text {even }}$ that is even with 
respect to $N$, the linear interpolation $L_{h} v_{\text {even }}$ is even on $N+\Pi_{h}$ (e.g., $N$ being a vertex or a midpoint of an edge of the uniform partition considered).

We now prove that for a quadratic polynomial $q$ we have $F_{i, N}(q)=0$ for $i=1, \ldots, d$. Decompose

$$
q(x)=q_{\text {even }}(x)+q_{\text {odd }}(x)
$$

into even and odd (with respect to the point $N$ ) polynomial parts; clearly $q_{\text {odd }}$ is linear, thus $q_{\text {odd }}=L_{h} q_{\text {odd }}$ and $F_{i, N}\left(q_{\text {odd }}\right)=0$. The local symmetry condition implies that $L_{h} q_{\text {even }}$ is an even function. Then both $\left(\partial / \partial x_{i}\right) q_{\text {even }}$ and $\left(\partial / \partial x_{i}\right) L_{h} q_{\text {even }}$ are odd functions and have zero mean over $N+\Pi_{h}$ (a set that is point-symmetric with respect to $N)$. Therefore, $F_{i, N}(q)=0$ for all quadratic functions and $i=1, \ldots, d$ (for all $d \geq 1$ ).

3.6. Dimension $d=2$. For $d=2$ the postprocessed function $S_{h} \nabla L_{h} u$ is piecewise quadratic (see Lemma 3.2). Since $S_{h} \nabla q=\nabla q$ is linear for a quadratic polynomial $q\left(S_{h} \nabla q\right.$ being the average of a linear function over an area symmetric with respect to its center of gravity) and $S_{h} \nabla q=S_{h} \nabla L_{h} q$ at the vertices and midpoints of edges of triangles, we have

$$
\nabla q=S_{h} \nabla q=S_{h} \nabla L_{h} q .
$$

We shall now follow a Bramble-Hilbert lemma like sequence of arguments. From (16) we see that

$$
\begin{aligned}
\left\|S_{h}\left(\nabla u-\nabla L_{h} u\right)\right\|_{0, \tau_{h}} & =\left\|S_{h}\left(\nabla(u-q)-\nabla L_{h}(u-q)\right)\right\|_{0, \tau_{h}} \\
& \leq C\left\|\nabla(u-q)-\nabla L_{h}(u-q)\right\|_{0, \tau_{h}+\Pi_{h}} \\
& \leq C^{\prime} h|u-q|_{2, \tau_{h}+\Pi_{h} .}
\end{aligned}
$$

Making use of results of approximation theory (see [3]), we get

$$
\inf _{q}|u-q|_{2, \tau_{h}+\Pi_{h}} \leq C h|u|_{3, \tau_{h}+\Pi_{h}}
$$

and consequently,

$$
\left\|S_{h}\left(\nabla u-\nabla L_{h} u\right)\right\|_{0, \tau_{h}} \leq C h^{2}|u|_{3, \tau_{h}+\Pi_{h}} .
$$

Summing over all triangles $\tau_{h}$, we get

$$
\begin{aligned}
\left|S_{h}\left(u-L_{h} u\right)\right|_{1, \Omega_{0}} & =\sqrt{\sum_{\tau_{h}}\left\|S_{h}\left(\nabla u-\nabla L_{h} u\right)\right\|_{0, \tau_{h}}^{2}} \\
& \leq C h^{2} \sqrt{\sum_{\tau_{h}}|u|_{3, \tau_{h}+\Pi_{h}}^{2}} \leq C^{\prime} h^{2}|u|_{3, \Omega} .
\end{aligned}
$$

Putting (12), (13), and (17) together, we finally prove the bound

$$
\left\|u-S_{h} u_{h}\right\|_{1, \Omega_{0}} \leq C h^{2}\|u\|_{3, \Omega}
$$

for $u \in H^{3}(\Omega)$. 
3.7. Superconvergence for the diagonal directional derivative $\sum_{i=1}^{d} \partial / \partial x_{i}$. Before we look into the general case of arbitrary space dimension, we shall take a closer look at the case of the diagonal derivative.

The postprocessed function $S_{h}\left(\sum_{i=1}^{d} \frac{\partial}{\partial x_{i}} L_{h} u\right)$ has a simpler structure than the functions $S_{h}\left(\frac{\partial}{\partial x_{i}} L_{h} u\right), i=1, \ldots, d$, have, allowing a simple proof of a superconvergence result for the diagonal derivative.

For an edge $e$ of the simplicial partition in the direction $(1, \ldots, 1) \in \mathbb{R}^{d}$, the patch of simplices sharing this edge is a $d$-hypercube with $e$ being its diagonal (see [2]). Furthermore, the derivative along $e$ is constant on the $d$-hypercube. Now clearly $S_{h}\left(\sum_{i=1}^{d} \frac{\partial}{\partial x_{i}} L_{h} u\right)$ is piecewise linear in each direction $e_{i} \in \mathbb{R}^{d}$ with $j$ th component $e_{i j}=\delta_{i j}$, so it is a piecewise linear tensor product function (e.g., piecewise bilinear for $d=2$ ).

The bound

$$
\left\|S_{h} \sum_{i=1}^{d} \frac{\partial}{\partial x_{i}}\left(u-L_{h} u\right)\right\|_{0, \Omega_{0}} \leq C h^{2}\|u\|_{3, \Omega}
$$

is now apparent, since similarly to (16) we have

$$
\sum_{i=1}^{d} \frac{\partial}{\partial x_{i}} q=S_{h} \sum_{i=1}^{d} \frac{\partial}{\partial x_{i}} q=S_{h} \sum_{i=1}^{d} \frac{\partial}{\partial x_{i}} L_{h} q
$$

for any quadratic polynomial $q$.

3.8. Dimension $d \geq 3$. We shall now use the results for $d=2$ to prove the quadratic polynomial gradient recovery property (16) for general $d \geq 3$.

For $q$ linear, we clearly have $L_{h} q=q$, and therefore, (16) is true.

It remains to prove (16) for quadratic monomials

$$
q(x)=x_{i} x_{j}, \quad i, j \in\{1, \ldots, d\} .
$$

Fix $i, j$. For an integer $k \notin\{i, j\}$, the monomial $q$ does not depend on $x_{k}$ and so $\partial q / \partial x_{k}$ is zero. The derivative $\left(\partial / \partial x_{k}\right) L_{h} q$ is constant on each $d$-simplex and is determined by the values of $L_{h} q$ at the vertices of the edge in the canonical direction $e_{k}$. Each $d$-simplex of the partition considered has such an edge (see [2]). Therefore,

$$
\frac{\partial}{\partial x_{k}} q=\frac{\partial}{\partial x_{k}} L_{h} q=0 \quad \text { for } k \notin\{i, j\},
$$

and thus,

$$
S_{h} \frac{\partial}{\partial x_{k}} L_{h} q=0 \quad \text { for } k \notin\{i, j\} .
$$

We shall now investigate the case of the $\partial / \partial x_{i}$ derivative. Setting $\Pi_{h}^{m}=$ $(-h, h)^{m}$ and taking a permutation $\sigma$ of $1, \ldots, d$ such that $\sigma(d-1)=i$ and 
$\sigma(d)=j$, we have

$$
\begin{aligned}
S_{h} \frac{\partial}{\partial x_{i}} L_{h} q & =\frac{1}{(2 h)^{d-2}} \int_{\Pi_{h}^{d-2}}\left(\frac{1}{(2 h)^{2}} \int_{\Pi_{h}^{2}} \frac{\partial}{\partial x_{i}} L_{h} q d x_{i} d x_{j}\right) d x_{\sigma(1)} \cdots d x_{\sigma(d-2)} \\
& =\frac{1}{(2 h)^{2}} \int_{\Pi_{h}^{2}} \frac{\partial}{\partial x_{i}} L_{h} q d x_{i} d x_{j},
\end{aligned}
$$

since $L_{h} q$ does not depend on $x_{\sigma(1)}, \ldots, x_{\sigma(d-2)}$.

It now follows from (16) for the two-dimensional case that $S_{h}\left(\partial / \partial x_{i}\right) L_{h} q$ is a linear function (depending on $x_{i}$ and $x_{j}$ only).

Therefore, (16) is proven for $d \geq 3$. Following an identical sequence of arguments as for the two-dimensional case, we come to the bound (10) of Theorem 3.1.

Having worked so far with functions $u \in H^{s}(\Omega)$ (with $s=3$ when $d \leq 5$ and $s>d / 2$ when $d \geq 6$ ), we shall now complete the proof for functions $u \in H^{3}(\Omega)$ by a density argument.

Let $u \in H^{3}(\Omega)$ and $\varepsilon>0$ be given. By density of $H^{s}(\Omega)$ in $H^{3}(\Omega)$ there exists a function $w \in H^{s}(\Omega)$ such that $\|u-w\|_{3, \Omega} \leq \varepsilon$. By the triangle inequality

$$
\begin{aligned}
\left\|u-S_{h} u_{h}\right\|_{1, \Omega_{0}} & \leq\left\|(u-w)-S_{h}\left(u_{h}-w_{h}\right)\right\|_{1, \Omega_{0}}+\left\|w-S_{h} w_{h}\right\|_{1, \Omega_{0}} \\
& \leq C \varepsilon+C h^{2}\|w\|_{3, \Omega} \leq C^{\prime} \varepsilon+C h^{2}\|u\|_{3, \Omega},
\end{aligned}
$$

where $w_{h}$ is the Galerkin projection of $w$ (corresponding to the boundary value problem considered and the finite element space used). Since $\varepsilon>0$ was arbitrary, we arrive at

$$
\left\|u-S_{h} u_{h}\right\|_{1, \Omega_{0}} \leq C h^{2}\|u\|_{3, \Omega}
$$

for $u \in H^{3}(\Omega)$.

3.9. Pointwise estimates at local symmetry points. Estimates at a symmetry point $N$ yield

$$
\left|F_{i, N}(u)\right| \leq \frac{C}{(2 h)^{d}}\left\|\frac{\partial}{\partial x_{i}}\left(u-L_{h} u\right)\right\|_{0, N+\Pi_{h}}\|1\|_{0, N+\Pi_{h}} \leq \frac{C^{\prime}}{(2 h)^{d / 2}} h|u|_{2, N+\Pi_{h}}
$$

and since $F_{i, N}(q)=0$ for all quadratic polynomials $q$, we can write

$$
\left|F_{i, N}(u)\right| \leq \frac{C}{(2 h)^{d / 2}} h|u-q|_{2, N+\Pi_{h}} .
$$

The use of approximation theory (see [3]) then yields the following result, which we will employ later in Section 3.11. 
Lemma 3.3. There exists a constant $C>0$ such that if $N$ is a symmetry point of the mesh, then

$$
\left|F_{i, N}(u)\right| \leq \frac{C}{(2 h)^{d / 2}} h^{2}|u|_{3, N+\Pi_{h}}, \quad i=1, \ldots, d,
$$

for $h$ small enough.

3.10. Modifications. We can alter the above approach for $d=2$ to perform the averaging over a different area symmetric with respect to its center of gravity, e.g., $\Xi_{h}$ or $\Lambda_{h}$ of Figure 2, and obtain the same postprocessing accuracy as for the area $\Pi_{h}$. The proof would be identical as in the case of $\Pi_{h}$ as long as we choose to average over a point-symmetric area such that the results of Lemma 3.2 are valid.

Whereas results of Section 3.9 carry over to the case of a general pointsymmetric averaging area for $d \geq 3$, the condition of symmetry itself will not allow a similar proof of the quadratic polynomial recovery property (16) as in Section 3.8. However, (19) can still be utilized for proving error bounds of various averaging schemes as in the following section.
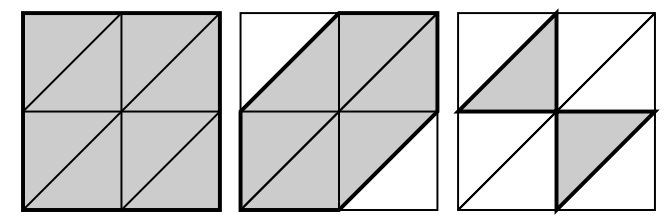

Fig. 2. Averaging areas $\Pi_{h}, \Xi_{h}$ and $\Lambda_{h}$

3.11. Gradient superconvergence. For arbitrary space dimension $d$ we easily obtain

$$
\begin{aligned}
& \left\|\nabla u-L_{h} S_{h} \nabla u_{h}\right\|_{0} \\
& \quad \leq\left\|\nabla u-L_{h} S_{h} \nabla u\right\|_{0}+\left\|L_{h} S_{h}\left(\nabla u-\nabla L_{h} u\right)\right\|_{0}+\left\|L_{h} S_{h}\left(\nabla L_{h} u-\nabla u_{h}\right)\right\|_{0} \\
& \quad \leq C h^{2}\|u\|_{3}+\left\|L_{h} S_{h}\left(\nabla u-\nabla L_{h} u\right)\right\|_{0},
\end{aligned}
$$

and employing the bound (19) at vertices to bound the remaining linear term, we easily get

$$
\left\|\nabla u-L_{h} S_{h} \nabla u_{h}\right\|_{0, \Omega_{0}} \leq C h^{2}\|u\|_{3, \Omega} .
$$

Choosing to average over $\Xi_{h}(d=2$, see Figure 2), one immediately gets the superconvergence result for the linear field (see [5])

$$
L_{h} S_{h} \nabla u_{h}(N)=\left.\frac{1}{6} \sum_{\tau \cap N \neq \emptyset} \nabla u_{h}\right|_{\tau},
$$

for $d=3($ see $[4])$ 


$$
L_{h} S_{h} \nabla u_{h}(N)=\left.\frac{1}{24} \sum_{\tau \cap N \neq \emptyset} \nabla u_{h}\right|_{\tau}
$$

or similarly for an arbitrary $d$ with coefficient $1 /(d+1)$ !.

Averaging over $\Lambda_{h}$ for $d=2$ leads to the postprocessing scheme (see [2])

$$
\begin{aligned}
& L_{h} S_{h} \partial_{x_{1}} u_{h}(N)=\frac{u_{h}(N+(h, 0))-u_{h}(N-(h, 0))}{2 h}, \\
& L_{h} S_{h} \partial_{x_{2}} u_{h}(N)=\frac{u_{h}(N+(0, h))-u_{h}(N-(0, h))}{2 h} .
\end{aligned}
$$

REMARK 3.4. The postprocessed solution $S_{h} u_{h}$ is not better in the $L^{2}$ sense, i.e., we only have

$$
\left\|u-S_{h} u_{h}\right\|_{0, \Omega_{0}}=\mathcal{O}\left(h^{2}\right),
$$

and this estimate cannot be improved.

4. Numerical experiments. We tested the superconvergence rate for the problem (8) with $A=I$ on the cube $\bar{\Omega}=[0,1]^{3}$. The first inequality in (11) allowed us to restrict ourselves to testing the second order of convergence of the interpolation $\left\|u-S_{h} L_{h} u\right\|_{1, \Omega}$ only. Results reported in Table 1 are for the function $u(x)=\prod_{i=1}^{3} \sin \left(\pi x_{i}\right)$, which is an eigenfunction of the problem considered. Note that in this case

$$
\left\|\partial_{1} u-S_{h} \partial_{1} L_{h} u\right\|_{0}>\left\|\sum_{i=1}^{3} \partial_{i} u-S_{h} \sum_{i=1}^{3} \partial_{i} L_{h} u\right\|_{0},
$$

as reported in Table 1. This observation is in agreement with Section 3.7.

The computation of the error in $L^{2}$-norms was performed with HIntLib, a $\mathrm{C}++$ package for high dimensional numerical integration.

The smoothening effect of the Steklov operator applied to two-dimensional continuous piecewise linear functions is shown in Figures 3 and 4.
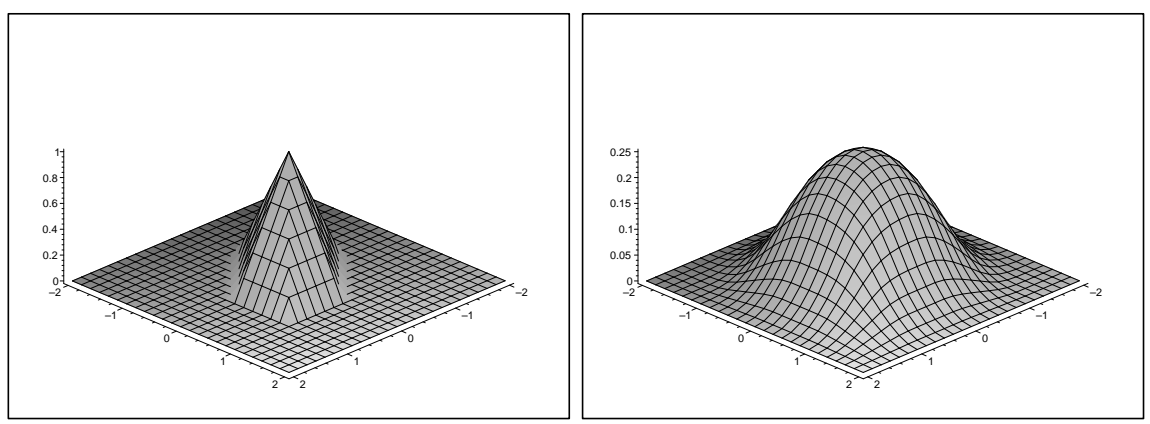

Fig. 3. Basis function for piecewise linear elements and its postprocessing 
Table 1. Reduction factors for $u=\prod_{i=1}^{3} \sin \left(\pi x_{i}\right)$

\begin{tabular}{ccccc}
\hline $1 / h$ & $\left\|\partial_{1} u-S_{h} \partial_{1} L_{h} u\right\|_{0}$ & reduct. & $\left\|\sum_{i=1}^{3} \partial_{i} u-S_{h} \sum_{i=1}^{3} \partial_{i} L_{h} u\right\|_{0}$ & reduct. \\
\hline 4 & 0.290806 & - & 0.1440776 & - \\
8 & 0.0997422 & 2.90 & 0.0485776 & 2.91 \\
16 & 0.029411 & 3.19 & 0.0152295 & 3.39 \\
32 & 0.00785763 & 3.32 & 0.00458811 & 3.74 \\
\hline
\end{tabular}
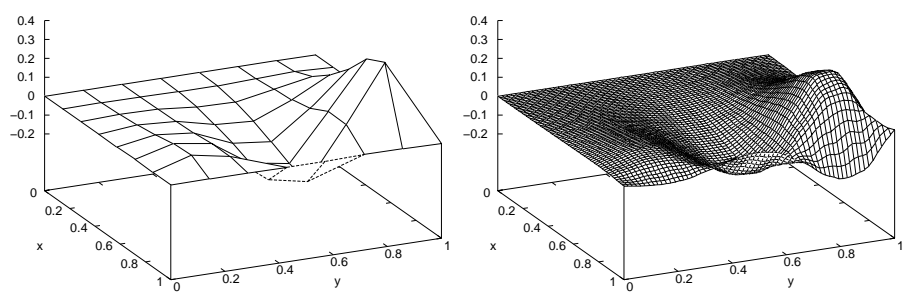

Fig. 4. Graphs of $L_{h} u$ and $S_{h} L_{h} u$ for $u\left(x_{1}, x_{2}\right)=e^{-2 / \sqrt{\vartheta}} \exp \left(\left(b_{1} x_{1}+b_{2} x_{2}\right) / 2 \vartheta\right)$. $\sin \left(k \pi x_{1}\right) \sin \left(l \pi x_{2}\right)$ with $\vartheta=1 / 10, b_{1}=1, b_{2}=0, k=1, l=3$ for $h=1 / 8$ (graph of $S_{h} L_{h} u$ fitted to an $h^{2}$ mesh)

Acknowledgements. This paper was supported by Grant No. A 1019201 of the Grant Agency of the Academy of Sciences of the Czech Republic.

\section{References}

[1] J. H. Bramble and A. H. Schatz, Higher order local accuracy by averaging in the finite element method, Math. Comp. 31 (1977), 94-111.

[2] J. Brandts and M. Kř́žek, Gradient superconvergence on uniform simplicial partitions of polytopes, IMA J. Numer. Anal. 23 (2003), 489-505.

[3] S. C. Brenner and L. R. Scott, The Mathematical Theory of Finite Element Methods, Springer, New York, 2002.

[4] V. Kantchev and R. D. Lazarov, Superconvergence of the gradient of linear finite elements for $3 D$ Poisson equation, in: Proc. Internat. Conf. Optimal Algorithms, B. Sendov (ed.), Blagoevgrad, Izd. Bulg. Akad. Nauk, Sofia, 1986, 172-182.

[5] M. Kř́žzek and P. Neittaanmäki, Superconvergence phenomenon in the finite element method arising from averaging gradients, Numer. Math. 45 (1984), 105-116.

[6] L. A. Oganesyan and L. A. Rukhovets, Variational-Difference Methods for the Solution of Elliptic Equations, Izd. Akad. Nauk Armyanskoŭ SSR, Erevan, 1979.

[7] R. Schürer, HIntLib, High-dimensional Integration Library, http://www.cosy.sbg. ac.at/ rschuer/hintlib/

Mathematical Institute

Academy of Sciences of the Czech Republic

Žitná 25

11567 Praha 1, Czech Republic

E-mail: kolman@math.cas.cz

Received on 12.4.2005;

revised version on 10.10.2005 